\title{
TOPOLOGICAL ANALYSIS OF MU-TRANSPOSITION
}

\author{
SOOJEONG KIM \\ ${ }^{1}$ University College, Yonsei University, SeOul 120-749, Republic of Korea \\ E-mail address: soojkimeyonsei.ac.kr
}

\begin{abstract}
An $n$-string tangle is a three dimensional ball with $n$-strings which are properly embedded in the ball. In early 90's, C. Ernst and D. Sumners first used a tangle to describe a DNA-protein complex. In this model, DNA is represented by a string and protein is represented by a ball. $\mathrm{Mu}$ is a protein which binds to DNA at three sites and a DNA-Mu complex is called Mu-transpososome. Knowing the DNA topology within Mu-transpososome is very important to understand DNA transposition by Mu protein. In 2002, Pathania et al. determined that the DNA configuration within the Mu transpososome is three branched and five noded [12]. In 2007, Darcy et al. analyzed this by using mathematical tangle and concluded that the three branched and five noded DNA configuration is the only biologically reasonable solution [4]. In this paper, based on the result of Pathania et al. and Darcy et al., the author determines the DNA topology within the DNA-Mu complex after the whole Mu transposition process. Furthermore, a new experiment is designed which can support the Pathania et al.'s result. The result of this new experiment is predicted through mathematical knot thory.
\end{abstract}

\section{INTRODUCTION}

In mathematics, a knot is a string whose two ends are attached to each other to form a loop. One can think the string has no thickness and has no self-intersection in space [1]. We say two knots are same if one knot can be changed to the other by deformation. The simplest knot is an unknot which is just a circle and the next simplest knot is a trefoil knot (Figure 7). If $n$ many $(n>1)$ knots are tangled up together, we call it a link with $n$ components. A knot (or link) can be drawn on a two dimensional plane and such a picture of a knot (or link) is called a projection of the knot (or link). If we draw a three dimensional ball around some parts of a knot (or a link), we can get some segments of string in the ball. Mathematically, we define an $n$-string tangle as a set $(B, t)$ where $B$ is a three dimensional ball and $t$ is a set of $n$ segments which are properly embedded in the ball.

DNA is a sequence of 4 repeated units (nucleotides); A, T, C and G. In 3-dimensional view, DNA looks like a long string. Bacterial DNA is in a circular form in usual, and large DNA molecules in higher organism are linear overall. Even though DNA exists in a liner form, it is generally constrained within some protein so two ends are not allowed to rotate. This

Received by the editors November 2 2012; Accepted February 222013.

2000 Mathematics Subject Classification. 57M25; 92C40; 92E10.

Key words and phrases. DNA topology, Knot, Link, Mu-transpososome, Transposition, 
kind of structure is called loop structure which is analogous to a circular form $[2,19]$. When a circular DNA binds to a protein, the conformation of the DNA-protein complex is simply a ball (protein) with a circular string (DNA) which is very similar to the shape of a tangle. A tangle model for DNA-protein complexes are introduced by C. Ernst and D. Sumners in late 80's (see Figure 15) and mathematical analysis of a tangle model is very useful for understanding biological activities of the complex [3, 4, 5, 6, 7, 9, 10, 15, 16, 20, 12, 17, 18].

In 20th century, DNA is revealed as a genetic object of almost of all organisms. All living things have evolved over a long period of time, but extremely slow in speed. Hence it's very easy to think that the DNA with genetic information is steady element. However, it's not true. There are DNA sequences which can transport themselves to other location within a genome, called transposable elements or transposons and this biological process is called a transposition [11]. The reason why transposons are interesting is not only the mechanisms involved in the manipulation of DNA, but also the evolutionary consequences of their mobility. Maybe they provide the major source of mutations in the genome [11]. Thus, it is revealed that $45 \%$ of recent human genome sequence is composed of transposons [8].

There are proteins involved in transposition. Mu is the most efficiently joining protein in this activity. During the Mu transposition process(see Figure 6), Mu proteins bind to DNA at three sites including Enhancer, attL and attR. This DNA-Mu complex is called transpososome. Knowing the structure of DNA within the Mu proteins in this complex is very important to understand the whole transposition process[13]. In 2002, Pathania et al. predicted the 3branched 5-noded configuration of DNA within the transpososome[12]. In 2007, Darcy et al. used a 3-string tangle theory to confirm that this is the only biologically reasonable DNA topology within the Mu-DNA complex[4].

In this paper, the author reviews the process of Mu-transposition determined DNA topology within Mu-DNA complex after the whole transposition process. The author also constructs idea of experiments that can support Pathania et al.'s experimental result. Furthermore, the result of new experiment is predicted by using knot theory. Some biological and mathematical background is introduced in section 2 and section 3. In section 4, Pathania et al.'s difference topology experiment and Darcy et al's mathematical analysis is reviewed. In section 5, topology of DNA within the Mu-DNA complex after the whole transposition process. A newly designed experiment is also introduced and the result is predicted. The summary of this paper is in section 6 .

\section{Mu-TRANSPOSITION}

In this section, biological background is reviewed to understand the topological model of Mu-transposition in section 4 and 5.

A three dimensional image of DNA looks like a long string. However, if we zoom in on DNA, it is double stranded Each strand consists of 4 repeated nucleotides (A, T, C, G) and the two strands are connected by hydrogen bond. At this time, $\mathrm{A}$ on a strand always binds to $\mathrm{T}$ on the other strand and C always binds to G. Transposable elements or a transposons are discrete 
DNA sequences in the genome that can move. In other words, they can carry themselves to other locations within the genome [11].

There are two kinds of transposons, regarding to the size of them: insertion sequences and composite sequences. Insertion sequences are the simplest type of transposons, the size is about 1000 base pairs. These sequences are named IS elements. The two ends of the IS element consist of short inverted sequences: usually the two copies of the sequences are very similar rather than identical. The common size of the inverted repeats is 9 base pairs. The process of the transposition of an IS element is well shown in the Figure 1. An IS element is inserted in a host DNA, and at the insertion site, a short DNA is duplicated. This specified short DNA sequence is called a target site. In Figure 1, the target site consists of ${ }_{T G C C T}^{A C G G A}$. A larger class of transposons are called composite elements. A composite element consists of a central region and two ends. The central region is named transposon markers and two ends are IS modules. The two IS modules are sometimes oriented in the same direction or more commonly in the inverted direction as in Figure 2. In some cases, IS modules are identical and sometimes they are closely related.

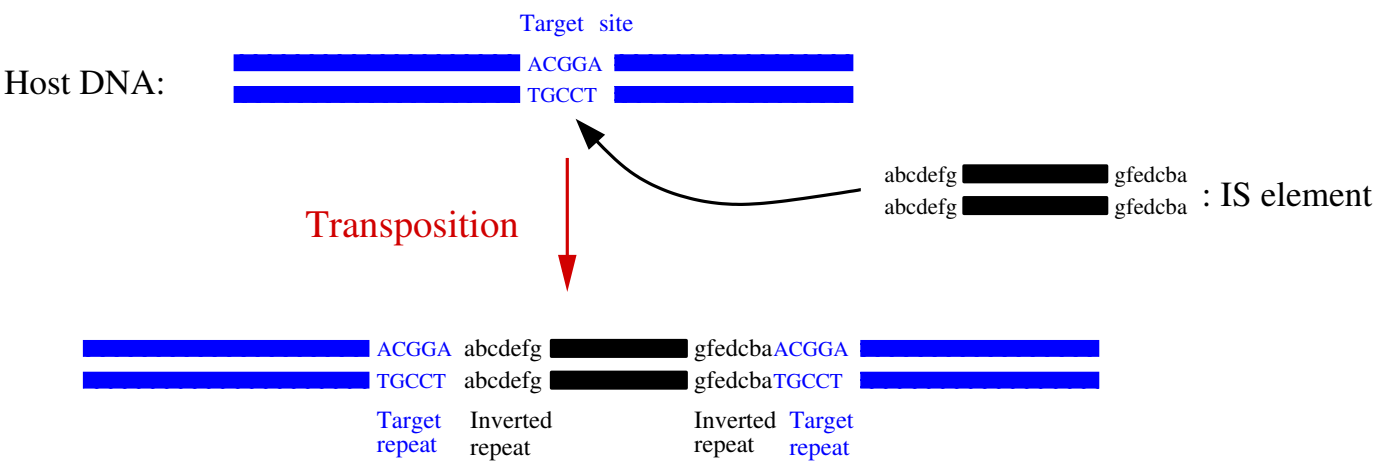

FIGURE 1. Transposition of an IS element. This figure is modified form [11]

The general mechanism of transposition is well illustrated in Figure 3. The first step is making staggered breaks in the DNA with target site. And then, by disconnecting the hydrogen bonds of target site, the target DNA will be divided into two segments with single stranded ends. The transposon is insulted between the two segment. The gaps between single stranded ends of target DNA and transposon are filled in and repaired.

All types of transpositions are commonly using the staggered ends(breaks). However, the transposition mechanism can be distinguished by three different types: replicative transposition, nonreplicative transposition and conservative transposition. During the transposition process, transposon element is transported from donor DNA to recipient DNA (or host DNA). In other words, the DNA which involves transposon element is called a donor DNA and the DNA with target sites of the transposon element is called recipient DNA. In replicative transposition, a transposable element is duplicated from the part of donor DNA (see Figure 4(a)) 
(a)

\begin{tabular}{|c|c|c|}
\hline 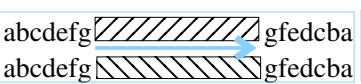 & Transposon markers & 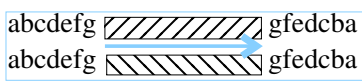 \\
\hline $\begin{array}{l}\text { IS module has } \\
\text { directly repeats }\end{array}$ & & $\begin{array}{l}\text { IS module has } \\
\text { directly repeats }\end{array}$ \\
\hline
\end{tabular}

(b)

\begin{tabular}{|c|c|c|}
\hline 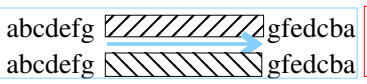 & Transposon markers & 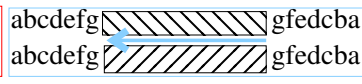 \\
\hline $\begin{array}{l}\text { IS module has } \\
\text { inverted repeats }\end{array}$ & & $\begin{array}{l}\text { IS module has } \\
\text { inverted repeats }\end{array}$ \\
\hline
\end{tabular}

FIGURE 2. Structure of Composite elements.

(a) Two arms of a composite element are directly oriented. (b) Two arms of a composite element are inversely oriented.

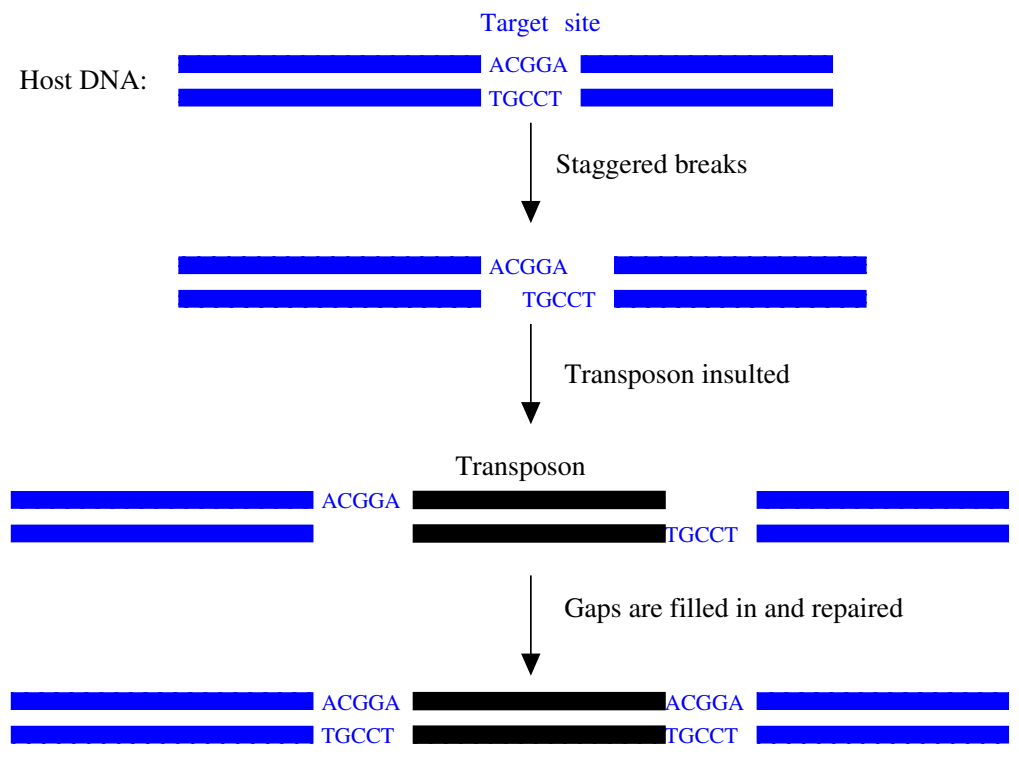

FIGURE 3. The general mechanism of transposition

and inserted into recipient DNA between two target sites. At the end of the replicative transposition, both donor DNA and recipient DNA have the same transposable elements. On the other hand, in nonreplicative transposition, the element form donor DNA moves directly to recipient DNA (see Figure 4(b)). The donor DNA is remained as broken two pieces and it can be destroyed or repaired. Especially, the case that the broken donor DNA is resealed in which 
all nucleotide bond is conserved is called conservative transposition (see Figure 4(c)). During transposition process, there are two types of enzymes involved:trasposase and resolvase. Transposase catalyze the transposition at the ends of the original transposon, and resolvase acts on the duplicated copies. Hence both enzymes are involved in replicative transposition and only transposase is involved in nonreplicative or conservative transposition.

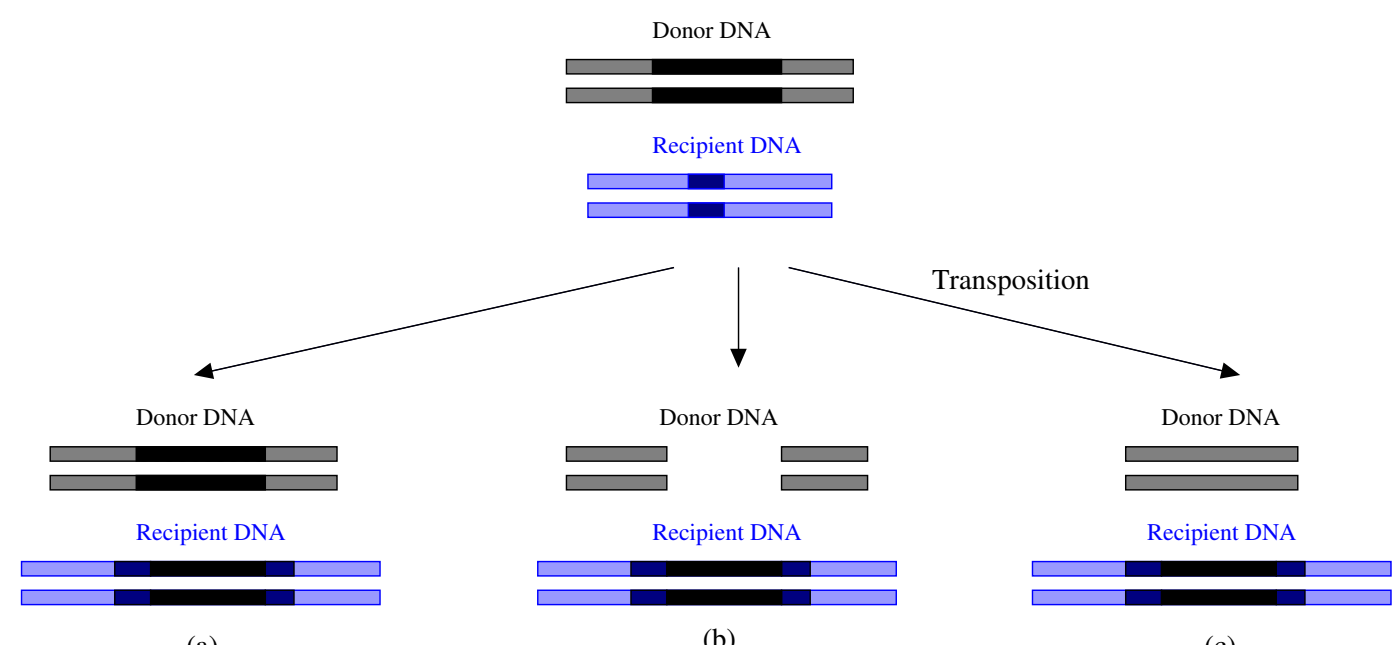

(a)

(b)

(c)

FIGURE 4. (a) Replicative transposition. (b) Nonreplicative transposition. (c) Conservative transposition.

$\mathrm{Mu}$ is a member of transposase which can help both replicative and nonreplicative transposition. We use the pathway of replicative transposition of $\mathrm{Mu}$ to predict a topological structure of Mu-DNA complex which is called Mu-transpososome. The basic pathway of replicative transposition is shown in Figure 5. In this figure, two stranded DNA is represented by single string. Donor DNA and recipient DNA (or host DNA) are circular DNA. By the assistance of a transposase, the nicked ends of transposon and staggered target sites are combined. The fusion product of a donor and recipient DNA is called a cointegrate.

The whole process of Mu transposition is well studied by Pathania et al. in [12] and [13]. There are two types of Mu proteins, MuA and MuB. Figure 6 shows the steps of Mu transposition: MuA binds to a circular DNA (donor DNA) involving the three DNA binding sites of $\mathrm{Mu}$ (enhancer, attL and attR sites), MuB captures a host DNA and combine it and the donor DNA.

\section{KNOTS/LINKS AND TANGLES}

Mathematical background is reviewed in this section to understand the topological model of Mu-transposition in section 4 and 5. 

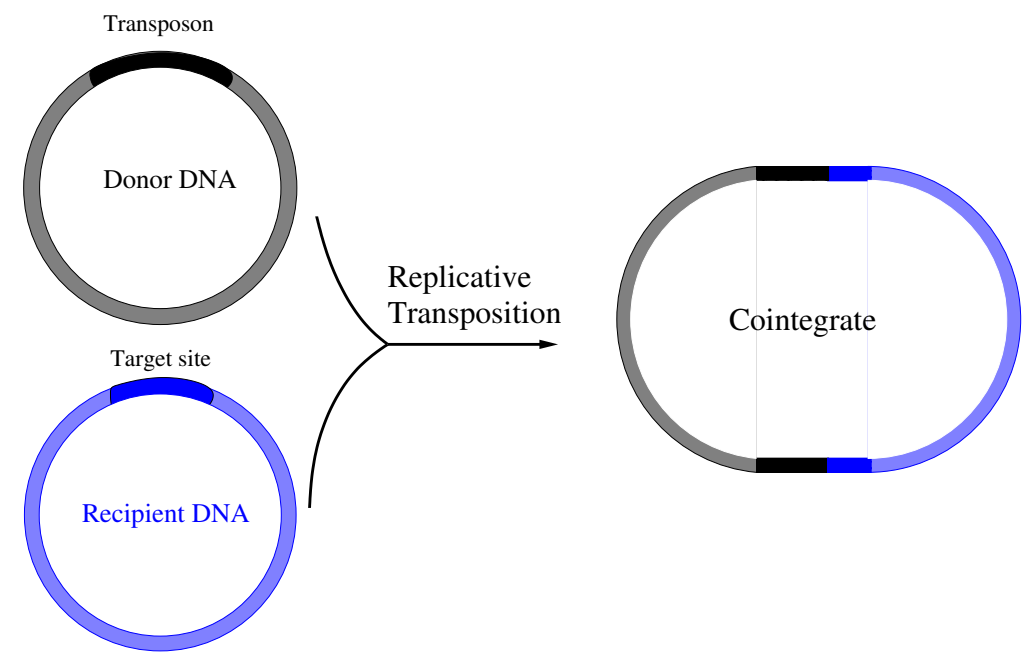

FIGURE 5. The basic route of replicative transposition. In this figure, two stranded DNA is represented by single string.

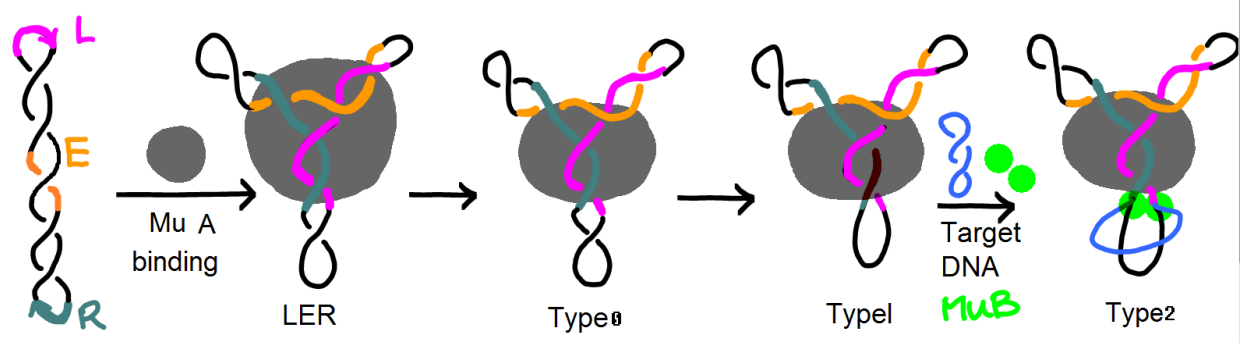

FIGURE 6. Mu Transposition. Figure is modified from [13]

3.1. Knots and links. A knot is a looped string without self-intersection in the three dimensional space. When there are more than one knotted loops which are all tangled up, we call it a link[1]. Knot or link can be defined with mathematical words as in Definition 3.1. The simplest knot is the unknotted circle which is called unknot (Figure 7(a)) and the next simplest knot is a trefoil knot which has three crossings (Figure 7(b)). The simplest link of two components with no crossing is the unlink or trivial link (Figure 7(c)) and the next simplest link of two components is the Hopf link which has two crossings (Figure 7(d)).

Definition 3.1. [14] A subset $K$ of a space $X$ is a knot if $K$ homeomorphic with a sphere $S^{p}$. More generally $K$ is a link if $K$ is homeomorphic with a disjoint union $S^{p_{1}} \cup \cdots \cup S^{p_{r}}$ of one or more spheres. 


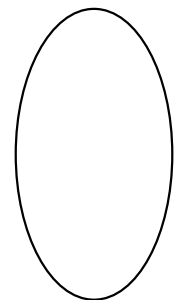

(a)

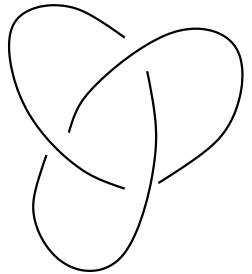

(b)

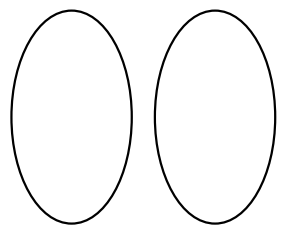

(c)

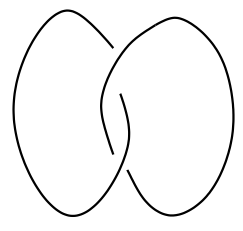

(d)

FIGURE 7. Examples of knots or links. (a) Unknot. (b) Trefoil. (c) Unlink. (d) Hopf link.

Two knots or links are same or equivalent if one can change a knot or a link to the other without cutting the string in the three dimensional space. In that case, we say two knots or links are isotopic and defined in Definition 3.2.

Definition 3.2. [14] Two knots or links $K, K^{\prime}$ in $X$ are isotopic if there is an ambient isotopy $F: X \times[0,1] \rightarrow X$ such that $F(K, 0)=K$ and $F(K, 1)=K^{\prime}$.

Eventhough knots or links are objects in the three dimensional space, it can be drawn on the two dimensional plane. A knot (or link) projection is a projection of a knot (or link) onto the 2-dimensional plane where under and over arcs are not specified. In this projection, no three arcs correspond to one point on the plane and arcs cross transversely. A knot (or link) diagram is a projection where at each crossing the over and under arcs are specified as in Figure 8 (a). If the diagram has an orientation, we can assign +1 or -1 at each crossing as in Figure 8(b), (c).
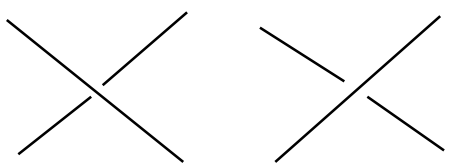

(a)

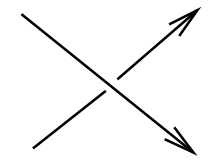

(b)

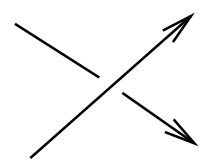

(c)

FIGURE 8. Crossings on a knot diagram

(a) Possible crossings on a knot diagram. (b) +1 crossing. (c) -1 crossing.

Two knot (or link) diagrams are equivalent if they are related by Reidemeister moves (Figure 9). The first move says that a twist can be put into or take out from the knot (or link) diagram as in Figure 9.I. The second move says that one can add or remove two crossings from the knot (or link) diagram as in Figure 9.II. The third move says that one can slide a strand of knot (or link) from one side of a crossing to the other side of the crossing as in Figure 9.III. Each of those three moves are ambient isotopy, hence two knot diagrams before and after Reidemeister moves are equivalent. 


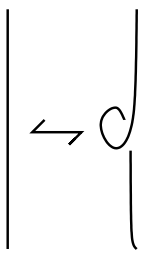

I

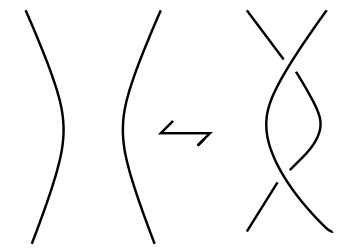

II

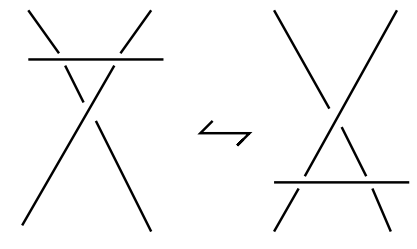

III

FIGURE 9. Reidemeister moves

Finally, two special knot types (used in section 5) are intoroduced in the following two definitions.

Definition 3.3. [1] A knot is called a torus knot if it lies on an unknotted torus, without crossing over or under themselves as they lie on the torus. When a torus knot wraps meridion of a torus p times and longitude or a torus q times, we say the torus knot as $(p, q)$-torus knot. (See Figure 10(a) for an example.)

Definition 3.4. [1] Let $K_{1}$ be a knot inside an unkntted solid torus. We knot that solid torus in the shape of a second knot $K_{2}$. This will take the knot $K_{1}$ that lies inside the original solid torus to a new knot inside the knotted solid torus. We call this new knot, $K_{3}$, a satellite knot. (See Figure 10(b) for an example.)

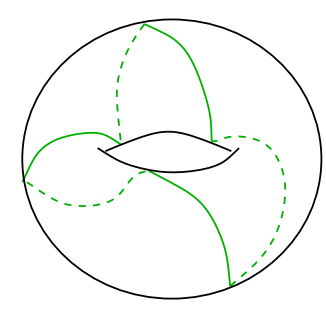

(a)

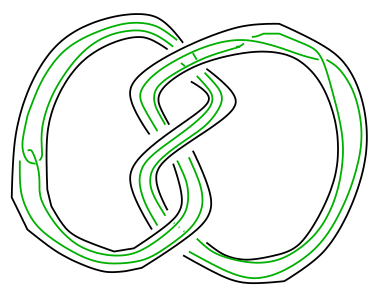

(b)

FiguRE 10. (a) Example of a (p, q)-torus knot. (b) Example of a satellite knot

3.2. Tangles. An n-string tangle is a three dimensional ball with $\mathrm{n}$ strings which are properly embedded in the ball (Definition 3.5). Examples of 3-string tangles are shown in Figure 11.

Definition 3.5. An n-string tangle is a pair $(B, t)$, where $B$ is a 3 dimensional ball and $t$ is a set of arcs embedded in $B$. The two end points of each arc lie on the boundary of $B$.

Two tangles are equivalent if they are ambient isotopic keeping the boundary of $B$ fixed. We say two tangles $T_{1}, T_{2}$ are freely isotopic if there is an isotopy of the 3-ball taking $T_{1}$ to $T_{2}$, which is not necessarily fixed on its boundary. 


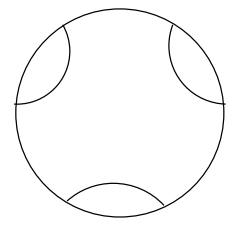

(a)

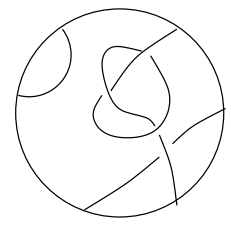

(b)

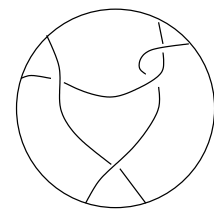

(c)

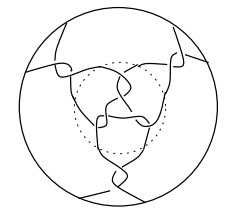

(d)

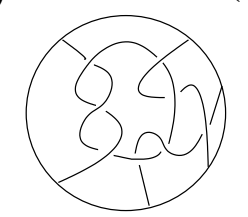

(e)

FIGURE 11. Examples of 3-string tangles

There are three types of tangle: If a tangle $T=(B, t)$ is freely isotopic to a tangle with no crossing, then we say $T$ is a rational tangle (for example, Figure 11(a), (c) and (d)). $T$ is locally knotted if there is a 2-sphere $S$ in $B$ that intersect one of the two strings transversely in two points and the string in $S$ is knotted with end points on $S$ (for example, Figure 11(b)). In the case that $T$ is neither rational nor locally knotted, $T$ is called a prime tangle (for example, Figure11(e)).

\section{Difference Topology Experiments of Mu-transpososome}

In section 2, we look over the DNA transposition by Mu proteins. As in Figure 6, MuA binds to a circular host DNA at Enhancer(E), attL(L) and attR(R) during the transposition process. Such Mu-DNA complex is called $M u$ transpososome. Knowing the topology of DNA within the $\mathrm{Mu}$ transpososome is very important key to understand the whole process of $\mathrm{Mu}$ transposition [12]. Pathania et al. developed a method to determine the DNA topology within the complex which is called difference topology.

In difference topology experiment[12], a protein Cre is used. Cre binds to DNA at two specific sites and performs DNA recombination by cut-and-rejoin action as in Figure 12. Assume there is a supercoiled circular DNA with two Cre binding sites. The topology of DNA after Cre recombination is unlink as in Figure 13. However, when protein binds to DNA and trapped some DNA supercoils, the DNA topology after Cre recombination is Hopf link as in Figure 13. By using this difference of DNA topology between two Cre recombination products, one can predict the conformation of DNA within the DNA-protein complex.

Since $\mathrm{Mu}$ has three DNA binding sites, $\mathrm{Mu}$ transpososome has three DNA loops out of $\mathrm{Mu}$ proteins (see Figure 6 and 15). Pathania et al. performed Cre recombination with those outside DNA loops. Since there are three outside loops, one can do three different Cre recombination on three different pairs of DNA loops. Figure 14 summarizes the main difference topology 


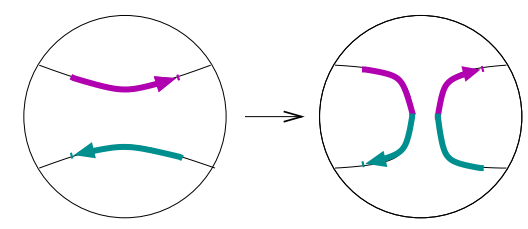

FIGURE 12. DNA recombination by Cre. Courtesy: I. Darcy.

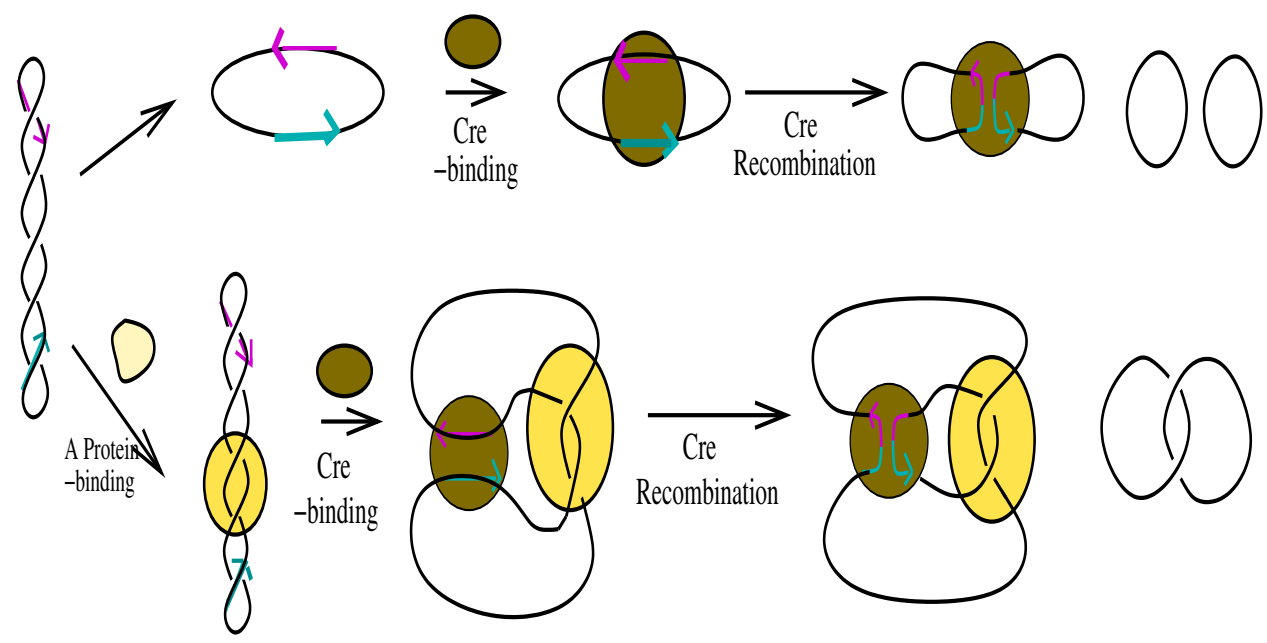

FIGURE 13. Example of difference topology experiments

experiments of $\mathrm{Mu}$ transpososome. In Figure 14, the gray ball represents $\mathrm{Mu}$ and the yellow ball represents Cre. In the first experiment, the product DNA of Cre recombination is a 3-noded knot (trefoil), which means there are three crossings between $\mathrm{E} \& \mathrm{~L}$ and $\mathrm{E} \& \mathrm{R}$. In the second experiment, the product DNA of Cre recombination is also a 3-noded knot (trefoil), which implies there are three crossings between $\mathrm{L} \& \mathrm{E}$ and $\mathrm{L} \& \mathrm{R}$. In the third experiment, the product DNA of Cre recombination is 4-noded link, which means there are four crossings between $\mathrm{R} \& \mathrm{E}$ and $\mathrm{R} \& \mathrm{~L}$. From putting all results together, one can conclude that the DNA topology within the $\mathrm{Mu}$ transpososome has 3-branched(this is biologically reasonable assumption [12]) five noded configuration such that two crossings between $E \& R$ and $L \& R$, one crossing between E \&L. Remark that Pathania et al. performed more experiments considering the orientation of Cre binding sites and linear DNA with Enhancer site. See [12, 13, 4, 9, 10,3] for more detail.

As one can see from Figure 6,12,13, and 14, a DNA-protein complex is simply drawn by a ball with strings. The ball represents a protein and the strings represent DNA. In the late 90's, D. Summers and C. Ernst developed a tangle model of a DNA-protein complex. This 

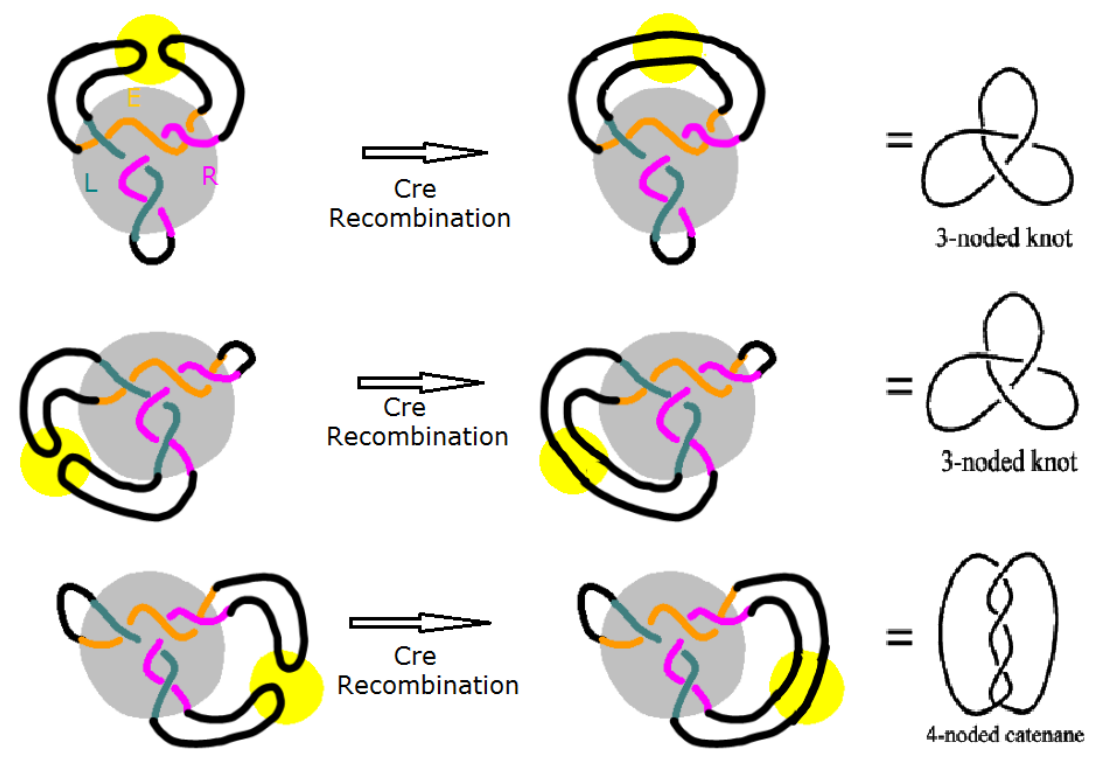

FIGURE 14. Difference topology experiments of Mu transpososome.

model assumes the protein is a three dimensional ball and the protein-bound DNA are strings embedded inside the ball as in Figure 15. Usually the main goal is knowing the tangle $T$, i.e. the shape of strings in the three dimensional ball of the tangle $T$ which represents the topology of protein bound DNA.
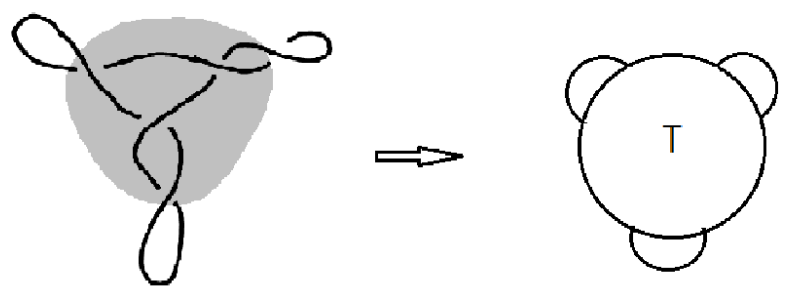

FIGURE 15. Mu transpososome corresponds to a 3-string tangle.

By using a tangle model, Pathania et al.'s difference topology experiments of Mu transpososome can be formulated as in Figure 16(a). In 2007, I. Darcy et al.[4] analyzed the 3-string tangle equations in Figure 16(a) and conclude that the only biologically relevant solution tangle is the tangle in Figure 16(b) which is congruent with Pathania et al.'s result. 


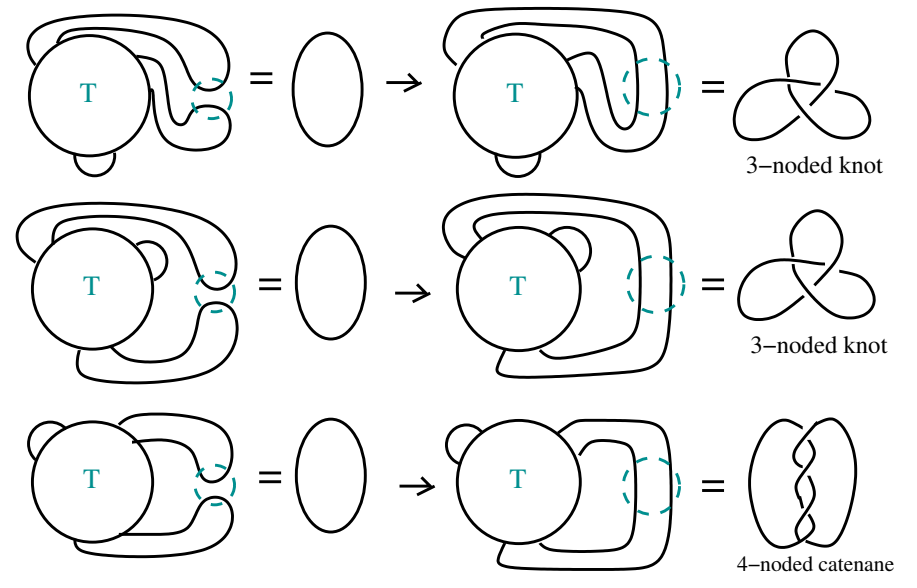

(a)

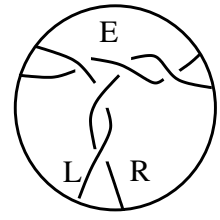

(b)

FIGURE 16. Tangle model of Mu transpososome. Figure from [9]

\section{DNA TOPOLOGY OF MU-TRANSPOSITION AND TyPE 2 COMPLEX}

In this section, we focus on the topology of DNA during the replicative transposition by $\mathrm{Mu}$. In other words, the topology of DNA within the 'type2' complex in Figure 6. While Pathania et al. used 'type 1' complex to determine DNA topology within the Mu and Darcy et al. analyzed a tangle model of it, the author suggest another different topology experiments with the 'type2' complex and discuss how this new method can support the existing result and theory.

As we've reviewed in section 2, replicative transposition of DNA proceeds trough a cointegrate (see Figure 5). The transposon involved in replicative Mu transposition is DNA segments which are composed of enhancer, attL and attR. There are two types of Mu proteins which are involved in this transposition, one is $\mathrm{MuA}$ and the other is $\mathrm{MuB}$. MuA is the protein that binds to DNA at three specific sites (enhancer, attL and attR) to form Mu-transpososome (type 1 complex in Figure 6) and activates site cleaves at the end of transposon. MuB protein captures the target DNA, recruits it to the active site of Mu A. Figure 17 illustrates the process of replicative DNA transposition by Mu proteins. To focus on the topology of DNA, the author does not mention about accessory factors(proteins) in transposition process (see [11, 12, 13] for more detail).

The topology of DNA after Mu transposition in Figure 17 stimulates curiosity about the topology of DNA within the 'type2' complex in Figure 6. Since the transposons are duplicated in a cointegrate product, applying the result in Figure 17 to type 2 complex is complicate and difficult work. Figure 18 is drawn very carefully by applying the product topology of $\mathrm{Mu}$ transposition to the conformation of type 2 complex. Note that Figure 18 (a) is topologically equivalent to the DNA topology of cointegrate product in Figure 17 as unlink. 


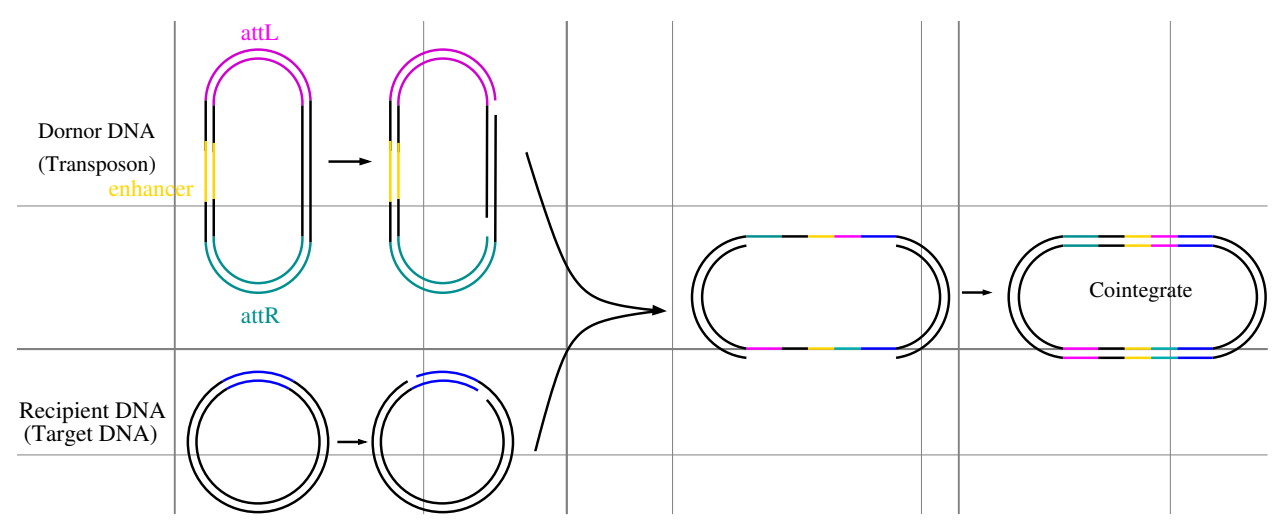

FIGURE 17. Replicative transposition process of DNA by Mu protein

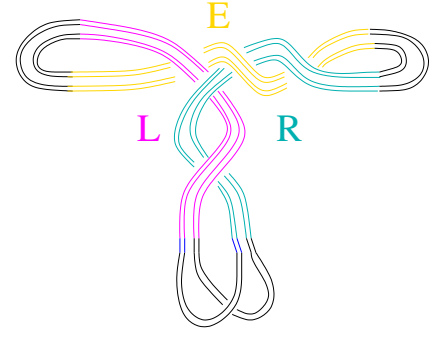

(a)

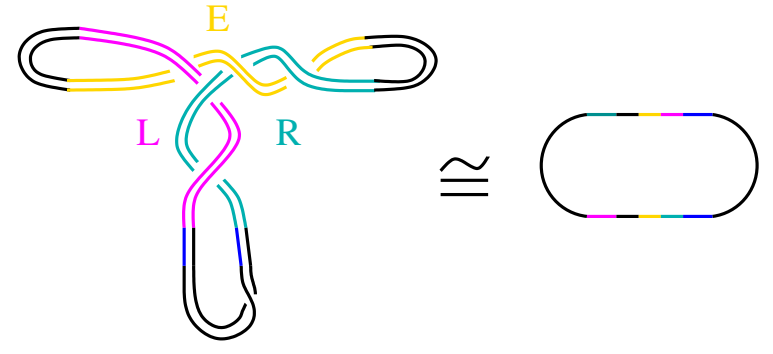

(b)

FIGURE 18. Topology of DNA within type 2 complex. In this figure, E, L and $\mathrm{R}$ represent enhaner, attL and attR, respectively. (a) Double strands of DNA are all drawn. (b) Double strands in part(a) is simplified to single string.

Our main goal is getting information about the conformation of Mu transpososome through the DNA topology of type 2 complex. As one can see from Figure 18 (b), when we regard two DNA strands as single string, the DNA topology within type 2 complex is unknot. This does not give us any new information, so one can consider performing cre recombination experiment before MuB protein captures target DNA. In other words, one can develop new difference topology experiment with following steps:

(1) Let MuA binds to a circular DNA involving the tree DNA binding sites, enhancer, attL and attR.

(2) When type 1 complex is obtained, perfom cre recombination on two outside DNA loops, call the product complex 'type $1^{*}$ '.

(3) MukB protein captures target DNA and let cointegrate product (type $1^{*}+\operatorname{target}$ DNA) be built through replicative transposition. Call the product complex 'type2*'. 
Of course, this experiment will be under the same environment with Pathania et al.'s experiment[12].

Since there are three different outside DNA loops on type1 complex, one can perfom three Cre recombination experiments on three pairs of two outside loops as in Figure 14 and so there are three different type $1^{*}$ complexes. MuB will capture target DNA and does transposition activity on each of three type $1^{*}$ complexes. Finally the three type $2^{*}$ complexes will be obtained. The schematic outline of this new difference topology experiment is shown in Figure 19.

(a)

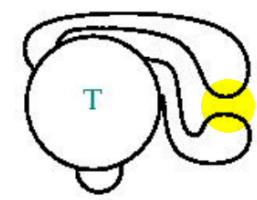

recombination

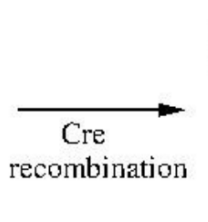

(b)
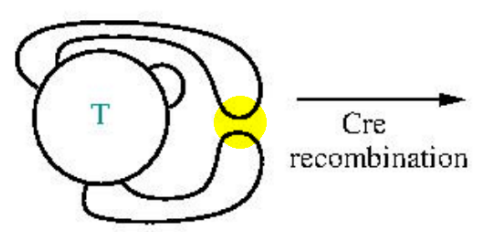

(c)

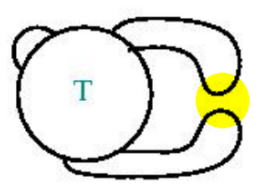

Type 1

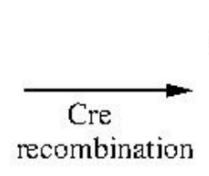

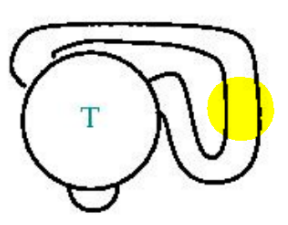
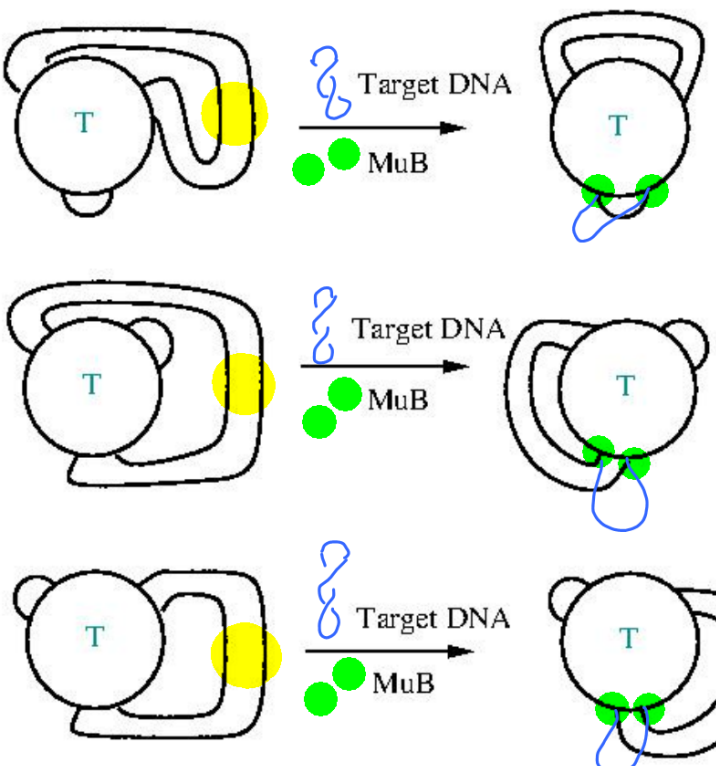

Type1*
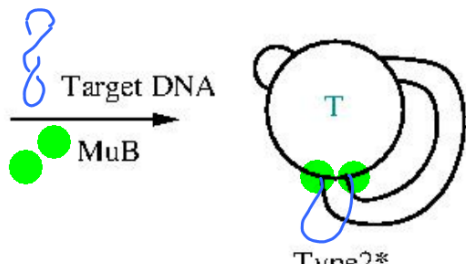

Type2*

FIGURE 19. Difference topology experiments with three different type $1^{*}$ complexes. In this figure, the yellow balls and green balls represent Cre and MuB protein, respectively.

Now, very interesting knots can be obtained as product DNA topology within type 2* complexes other than unknot. By applying the result of the topology of DNA within type 2 complex (Figure 18(b)), one can predict knot types of DNA within type2* complex. Let's get more into the experiment in Figure 19(a), then we can get a satellite knot with 13 crossings. See Figure 20. Similarly, for the experiments in Figure 19(b)\&(c), a (2,3)-torus knot and unknot are obtained by applying DNA topology within type2 complex in Figure 18(b). See Figure 21. As in Figure 21, the product DNA is much simpler than one in Figure 20. This is because, there appears a linear DNA during the process of constructing cointegrate products. A (2,p)-torus knots(or links) and unknot are very common knot types as DNA recombination product. 


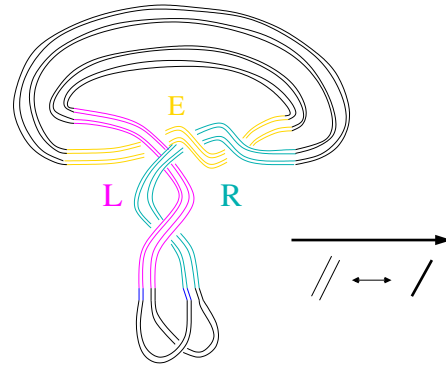

(a)

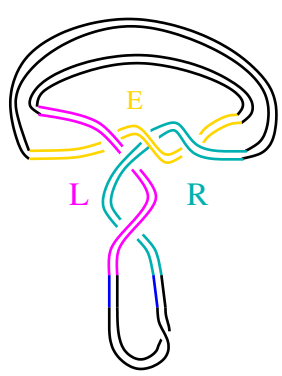

(b)

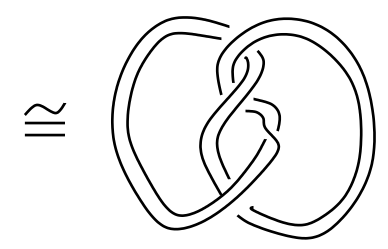

(c)

FIGURE 20. DNA topology within type2* complex which is in Figure 19(a). (a) Double stranded DNA are all drawn. (b) Double strands of DNA are simplified by single string. (c) A knot in part(b) is equivalent to a satellite knot with 13 crossings.

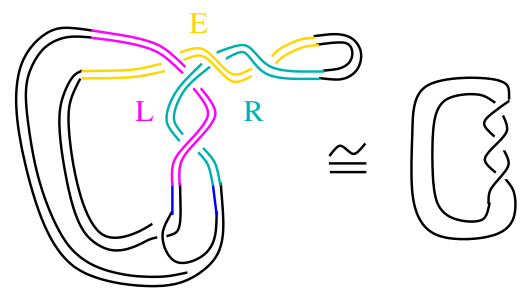

(a)

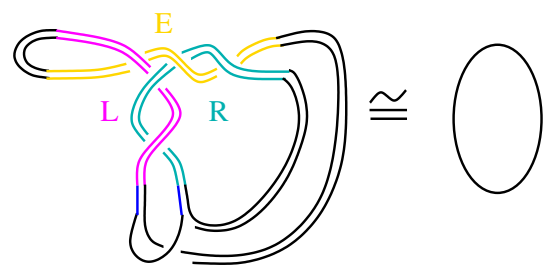

(b)

FIGURE 21. (a) DNA topology within type2* complex which is in Figure 19(b). The product DNA topology is equivalent to a $(2,3)$-torus knot. (b) DNA topology within type2* complex which is in Figure 19(c). The product DNA topology is unknot.

\section{SUMMARY}

In 2002, Pathania et al. determined that the conformation of DNA within Mu transpososome is three branched and five noded as in Figure 15. Based on this result[12], the author determined the DNA topology within type 2 complex. In other words, under the 3-branched and 5-noded assumption, the topology of DNA after replicative transposition by Mu is determined as in Figure 18. An experiment supporting Pathania et al.'s result is designed by means of DNA conformation within type 2 complex as in Figure 19. One can predict the product DNA topology of this new experiment as in Figure 20 and 21. Furthermore, if we get the result in real experiment, it can strongly support the result of Pathania et al.'s difference topology experiment: the 3-branched 5-noded DNA configuration within the Mu transpososome. 


\section{ACKNOWLEDGMENTS}

The author wishes to acknowledge the help of Dr. I. Darcy in deep discussion about this research. This research was supported by Basic Science Research Program through the NationalResearch Foundation of Korea(NRF) funded by the Ministry of Education, Scienceand Technology(2012R1A1A3015842).

\section{REFERENCES}

[1] C. Adams, The knot book: an elementary introduction to the mathematical theory of knots, American mathematical society, 1st ed., Providence, Rhode Island, 2004.

[2] A.D. Bates and A. Maxwell, DNA Topology, IRL Press Oxford, 1993.

[3] I. K. Darcy, A. Bhutra, J. Chang, N. Druivenga, C. McKinney, R. K. Medikonduri, S. Mills, J. Navarra Madsen, A. Ponnusamy, J. Sweet, and T. Thompson, Coloring the Mu transpososome, BMC Bioinformatics, 7:Art. No. 435 (2006).

[4] I.K. Darcy, J. Luecke, and M. Vazquez, Tangle analysis of difference topology experiments: Applications to a Mu protein-dna complex, Algebraic and Geometric Topology, 9 (2009), 2247-2309.

[5] F.B. Dean, A. Stasiak, T. Koller, and N.R. Cozzarelli, Duplex DNA knots produced by Escherichia Coli topoisomerase I, J. Biol. Chem., 260 (1985), 4795-4983.

[6] C. Ernst and D. W. Sumners, A calculus for rational tangles: applications to DNA recombination, Math. Proc. Camb. Phil. Soc., 108 (1990), 489-515.

[7] C. Ernst and D. W. Sumners, Solving tangle equations arising in a DNA recombination model, Math. Proc. Camb. Phil. Soc., 126 (1990), 23-36.

[8] International Human Genome Sequencing Consortium Initial sequencing and analysis of the human genome, Nature, 409 (2001), 860-921.

[9] S. Kim and I. Darcy, Topological Analysis of DNA-protein complexes, Mathematics of DNA Structure, function and interacctions, The IMA volumes in Mathematics and its applications, Springer Science + Business Media, LLC, New York, 2009

[10] S. Kim, A 4-string tangle analysis of DNA-protein complexes based on difference topology, $\mathrm{Ph} . \mathrm{D}$ thesis (2009)

[11] B. Lewin, Genes, Oxford University Press, 5th ed., New York, 1994.

[12] S. Pathania, M. Jayaram, and R. M. Harshey, Path of DNA within the Mu transpososome: transposase interactions bridging two Mu ends and the enhancer trap five DNA supercoils, Cell, 109(4) (2002), 425-436.

[13] S. Pathania, M. Jayaram, and R. M. Harshey, The Mu Transpososome Through a Topological Lens, Critical Reviews in Biochemistry and Molecular Biology, 41 (2006), 387-405.

[14] D. Rolfsen, Knots And Links, AMS Chelsea Publishing, Providence, Rhode Island, 1976.

[15] Y. Saka and M. Vazquez, Tanglesolve: topological analysis of site-specific recombination, Bioinformatics, 18 (2002), 1011-1012.

[16] S.J. Spengler, A. Stasiak, and N.R. Cozzarelli, The stereostructure of knots and catenanes produced by phage $\lambda$ integrative recombination : implications for mechanism and DNA structure, Cell, 42 (1985), 325-334.

[17] D. W. Sumners, C. Ernst, N.R. Cozzarelli, and S.J. Spengler, Mathematical analysis of the mechanisms of DNA recombination using tangles, Quarterly Reviews of Biophysics, 28 (1995).

[18] Alexandre A. Vetcher, Alexander Y. Lushnikov, Junalyn Navarra-Madsen, Robert G, Scharein, Yuri L. Lyubchenko, Isabel K. Darcy, and Stephen D. Levene, DNA topology and geometry in Flp and Cre recombination, J. Mol. Biol., 357 (2006), 1089-1104.

[19] A. V. Vologodskii Circular DNA. Annual Review of Biochemistry, John Wiley and Sons Inc, 40 (1971), 899942.

[20] S.A. Wasserman and N.R. Cozzarelli, Determination of the stereostructure of the product of Tn3 resolvase by a general method, Proc. Nat. Acad. Sci. U.S.A., 82 (1985), 1079-1083. 